\title{
MODELLING LONG-TERM MORPHODYNAMICS IN PRACTICE: UNCERTAINTIES AND COMPROMISES
}

\author{
J. J. WILLIAMS ${ }^{1}$, T. CONDUCHÉ ${ }^{1}$ AND L. S. ESTEVES ${ }^{2}$
}

1. Mott MacDonald, Mott MacDonald House, 8-10 Sydenham Road, Croydon, CRO 2EE,UK.jon.williams@mottmac.com; thomas.conduche@mottmac.com.

2. Faculty of Science and Technology, Bournemouth University, Talbot Campus, Poole, Dorset, BH12 5BB, UK. lesteves@bournemouth.ac.uk.

\begin{abstract}
Two problems limit the ability of morphological models to reproduce the real-world behaviour of geomorphological systems: (a) the dominance of short-term observations which fail to capture the full character of morphological evolution and cannot quantify fully the primary phenomena and mechanisms of change; and (b) incomplete understanding of processes at all relevant scales. Present efforts to reduce uncertainty in morphological models assume that: (a) observations are the key to locate, quantify and reduce uncertainty; and (b) means used to quantify, minimise and control uncertainty will improve model performance. This paper first outlines the principles underpinning morphological model development concerning coastal sandy environments. Then it discusses the use of morphological models and considers approaches that improve predictions through reduced uncertainty. The discussion is supported by two examples that illustrate the compromises that must be reached between what a morphological model is required to predict and what the model can deliver in practice.
\end{abstract}

\section{Introduction}

The ability of morphological models to reproduce the real-world behaviour of geomorphological systems is limited by two problems, which together restrain predictive accuracy and introduce uncertainty: (a) the dominance of short-term observations which fail to capture the full character of morphological evolution and cannot quantify fully the primary phenomena and mechanisms of change; and (b) incomplete understanding of processes at all relevant scales. At present the bulk of the effort to reduce uncertainty in morphological models is focused on finding better solutions to these problems and are based on the premise that: (a) observations are the key to locate, quantify and reduce uncertainty; and (b) means used to quantify, minimise and control uncertainty will improve model performance.

In nearly all cases, morphological models are concerned only with the physical environment. However, the typical linkages between various components of an idealised coastal system shown in Figure 1a demonstrate that ecological systems and human influences play a role in influencing coastal processes and evolution (e.g. Temmerman et al., 2013). It particular it is noted that geomorphological systems affect ecological systems and vice versa and both are influence by 
human actions (e.g. coastal engineering). With the exception of recent managed realignment studies ecological effects are rarely included in morphological models (e.g. Kirwan and Murray, 2007; Mossman et al., 2012) and land use changes, which can have significant impacts on sediment supply, are not considered. Although the magnitude to the impacts from these elements may differ according to the nature of the coastal system, their omission introduces errors and uncertainty in models that focus only on the geomorphological part of the system.

Morphodynamic models generally follow the pathways illustrated in Figure 1b. This shows links between hydrodynamics, sediment transport and morphology, data inputs and natural forcing and the feedback loop to update morphology in response to net sediment erosion or accretion. Figure $1 \mathrm{~b}$ shows also how errors arise at each node in the network and become compounded within the model structure owing to the non-linear nature of the processes. While data assimilation can reduce some of these errors (Figure 1b) it is only applicable in hindcast and nowcast studies.
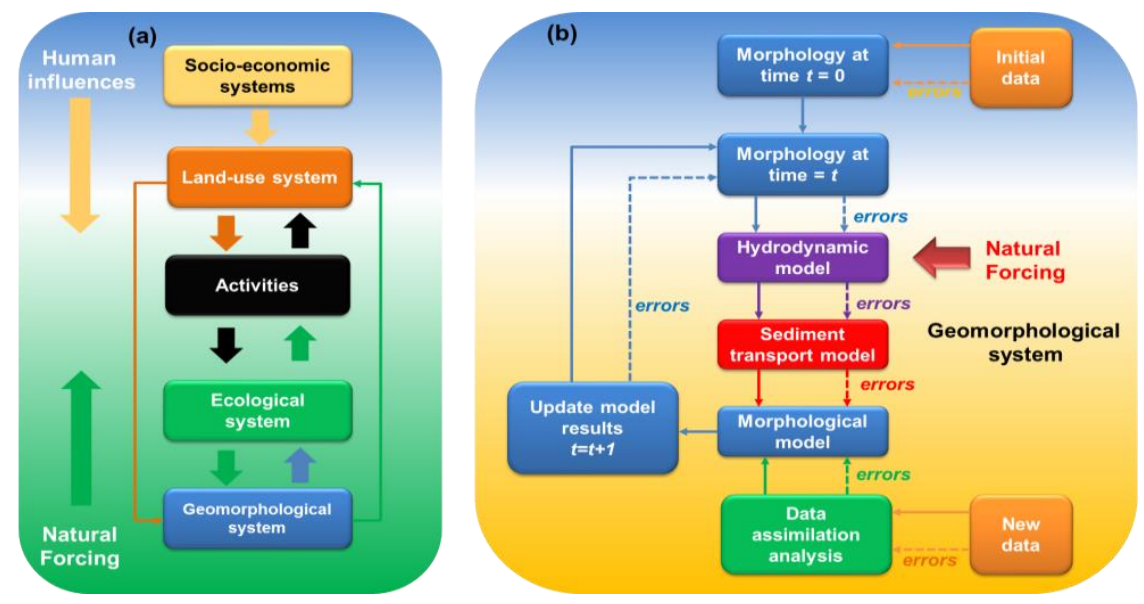

Fig. 1. (a) Relationships between natural and human-influenced coastal systems; and (b) typical morphological model setup including data assimilation.

For many practical applications concerned with, for example, coastal engineering design, scheme impacts or habitat creation by managed realignment, it is necessary to work with existing morphological models and to understand their limitations. This paper therefore first outlines the principals underpinning morphological model development before discussing the use of morphological models and considering approaches that improve predictions and reduce uncertainty. The discussion is supported by two examples that illustrate 
the compromises that must be reached between what a morphological model is required to predict and what the model can deliver in practice.

\section{General considerations and approach}

Before attempting morphological modelling of a coastal system it is useful to initially draw on all the available evidence and establish a conceptual understanding of local sediment transport and morphodynamic behaviour. This approach provides: (a) a hypothesis to test subsequent model performance; and (b) understanding of the expected magnitudes and directions of sediment transport and the associated rate and scale of morphological changes. In many practical applications, conceptual understanding will be as valuable as the results from morphological models and should not be overlooked.

If conceptual understanding identifies a need for morphological modelling, the following modelling principles must be considered: (1) Data and models are a limited representation of reality and models should always ensure available knowledge of the morphological system and model representation of forcing terms, processes and outputs are compatible (and the limits to the resolution of processes in models must be acknowledged); (2) from a practical perspective, forcing terms, processes and output cannot be determined beyond a certain timeand space-scale and models should not be pushed beyond these limitations; or put simply "crap data in equals equally crap model results out"; and (3) the amount of information in the model should be as close as possible to the original observations defining boundary conditions and processes; the aim should always be to neither gain nor loose information.

While a large number of parameters in a model can simplify the calibration process, they can also introduce greater uncertainty and increase the sensitivity of the model output to variations in the input (e.g. Turner et al., 1998). To prevent this, and to reduce model complexity per se, it is important also to limit the number of parameters in a model as far as practicable. It should be recognised that in all morphodynamic models, real-world complexity can only be handled in a simple way and the aim should be to model well the primary features, such as coastal cliff recession or spit progradation rather than reproduce the complex, potentially chaotic, dynamics. If features define the character of a system (e.g. bedforms), it is often easier to describe the behaviour of features rather than attempt to describe their formation and its behaviour together. This approach is used implicitly in theoretical morphodynamics to describe the occurrence of morphological patterns. 


\section{Improving predictions and reducing uncertainty}

In general two main types of uncertainty pervade morphological models: (a) scenario uncertainty stems from not knowing what the magnitude and frequency of future events will be or how present day sediment sources, pathways and sinks will change in the future; and (b) response uncertainty relates to the inherent uncertainty in morphological model prediction how a coastal system will respond to given forcing conditions. Uncertainty in morphodynamic models arises from three main sources: (1) the quality of the data defining boundary conditions (e.g. Figure 1b); (2) incomplete or inaccurate descriptions of the physical processes driving morphological changes; and to a lesser extent; and (3) limited computing power that constrains the temporal and spatial resolution of the model and the number of parameters used to describe processes.

To reduce uncertainty the ensemble approach widely adopted by climate change scientist is now being used increasingly by coastal modelers using the perturbed-physics approach to investigate how model predictions are affected by the choice of input parameters and through the use of multi-model studies to investigate how predictions differ between different models ( $c f$. Refsgaard et al., 2014) and how each model represent reality. The effect of the initial conditions of the model can also be tested using both approaches. However, even if the initial system state can be described flawlessly, model parameters simplify the physical processes and lead to the growth of prediction errors.

An alternative emerging approach to address the problem of model prediction uncertainties involves the application of data assimilation techniques (e.g. Smith et al., 2013; Mayo et al., 2014; Figure 1b). These techniques keep model parameters fixed and produce an updated model state that matches as closely as possible the true state by combining observational data with model predictions. This updated model state is then used to initiate the next model forecast. However, even if the initial morphology can be described accurately, model parameters simplify the physical processes and by doing so will result in the growth of prediction errors. At present assimilation methods being developed to improve morphological forecast reliability are producing encouraging results (e.g. Scott and Mason, 2007; Smith et al., 2013; Chu et al., 2013; Margvelashvili et al., 2013; Mayo et al., 2014). For example ad hoc data assimilation schemes and techniques using more refine heuristic tuning of model state variables are being used to improve the performance of suspended sediment transport models (e.g. Serafy et al., 2011; Margvelashvili and Campbell, 2012). A potentially useful way to reduce uncertainty is now emerging using hybrid morphological models that combine, for example, a deterministic 2D sediment transport model with a simplified morphological 
updating scheme in which the cross-shore profile is described using a limited number of parameters in a greatly simplified morphological scheme (e.g. Kristensen et al., 2013). However, even with the most effective data assimilation scheme, the projection of model prediction into the future remains limited and the testing of multiple scenarios remains the only effective means of addressing coastal evolution far into the future.

The sensitivity of a model to variations in parameters or data is of critical importance to morphological modelling and sensitivity analyses enable identification of where calibration and modelling efforts should be targeted to achieve the best outcome. Sensitivity analyses can be used to: (a) evaluate changes in model output due to changes in input variables; and (b) examine how uncertainty in individual model parameters can affect the overall model performance. Methods employed to test sensitivity and to quantify uncertainty include Monte Carlo analysis, fuzzy mathematics and Bayesian approaches. Irrespective of the approach used, it is important to establish: (a) the input variables to be tested; and (b) the optimum modelling strategy giving reliable results with the least number of simulations. Morphological models pose an additional problem when attempting to define sensitivity and uncertainty since non-linearity between processes frequently result in gross feature of the model output being relatively insensitive to variations in inputs until some morphological threshold is reached. Owing to incomplete descriptions of processes in a model such thresholds can cause large variations to occur from small modifications of the inputs (e.g. catastrophic behaviour) and may not be predicted by the model or detected by sensitivity analyses. To date this aspect of morphological modelling has been scarcely addressed.

An example of sensitivity analysis is shown in Figure 2 which shows part of a larger model built to examine the hydrodynamic and morphological impacts of a simple groyne. The initial bathymetry and structure, and a snapshot of the waveinduced alongshore current, are shown in Figures 2(a) and 2(b), respectively. Assuming the same median sediment grain size, $\mathrm{D}_{50}$, of $0.3 \mathrm{~mm}$ across the model domain, the predicted net erosion and accretion occurring during a month-long simulation using measured wave and tidal conditions (including a moderate storm) is shown in Figure 2(c). Clearly the assumption about the sediment distribution is a simplification of reality and in Figure 2(d), a grain size distribution that decreases in size from $\mathrm{D}_{50}=0.3 \mathrm{~mm}$ on the beach to $0.15 \mathrm{~mm}$ offshore direction is imposed. While these changes impact slightly on the predicted net erosion and accretion, little additional information about the overall impact of the groyne that might influence design decisions is evident. In this case, similar results were obtained in other model runs where the nature and spatial distribution of the bed sediments was changed thereby reducing the uncertainty in the model predictions and providing confidence that the proposed 
design would have the desired effect in promoting accretion for beach protection.
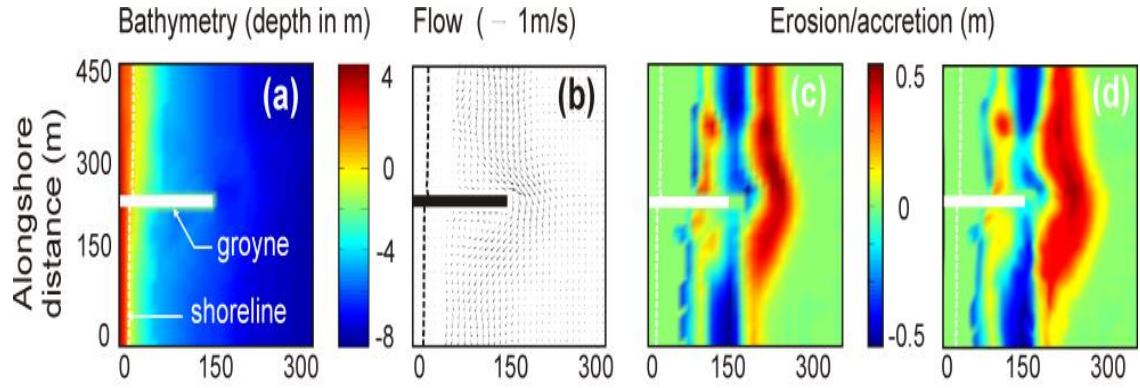

Cross-shore distance $(\mathrm{m})$

Fig. 2. Example of morphological model sensitivity: (a) emplacement of a groyne; (b) resulting flow vectors for tidal and wave forcing; (c) patterns of erosion and accretion using a spatially invariant median sediment grain size, $D_{50}$ of $0.3 \mathrm{~mm}$; (d) patterns of erosion and accretion using drag coefficient values based on bed sediment grain size.

In sensitivity analyses used to identify and quantify uncertainty in model predictions it is important to correctly identify the components of the model that have the greatest influence. For example, the correction of errors in the measured bathymetry during model calibration can be significantly more efficient than making adjustments to the bed roughness (e.g. Cea and French, 2012). Further, the temporal resolution of bathymetric data must reflect processes, with models of storm-dominated sites requiring shorter and more frequently sampled data sets and site dominated by seasonal forcing requiring monthly monitoring over several years (e.g. Splinter et al., 2013). In this respect reference to the conceptual model established at the start of any morphological modelling exercise can guide the modeler as to which aspects of a given model are most sensitive to errors in the boundary conditions.

\section{Compromises}

Inevitable in the real world, where prediction of future coastal evolution are required to inform engineering designs, to assess environmental impacts and for management and planning purposes to name but a few, a compromise must be reached between the end-user requirements for a given morphological model and the ability of that model to deliver the results required. Here we briefly discuss two recent studies. 


\section{Rossbeigh Spit, Ireland}

Rossbeigh Spit, located in Dingle Bay, Ireland was breached by a storm in 2008 (Figure 3). Predictions of the breaching event morphodynamics by XBeach demonstrated reasonably agreement with the observations (Williams et al., 2015). However, as is frequently the case in most coastal studies of this nature, the data describing the hydrodynamics, waves and the bathymetry and topography were incomplete and recourse to hindcast data and varied data sources to establish the pre- and post-storm bathymetry was required. Nevertheless, these difficulties were largely overcome and the main features of the breach were reproduced well by XBeach thus allowing determination of the site-specific critical wave and water level conditions giving rise to dune erosion, overwashing and breaching. By then deriving simple-to-use expressions to define hydrodynamic thresholds, the study advanced the ability to predict the impacts of infrequent and rarely observed storm events and is considered to provide useful coastal management tool for assessing the vulnerability of sandy barriers to breaching high-energy during storms.

(a)

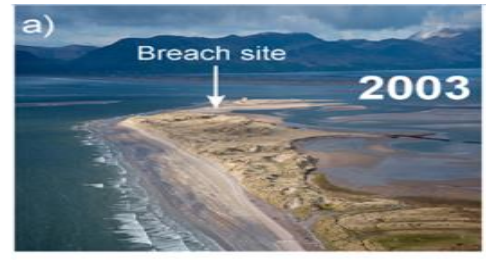

(c)

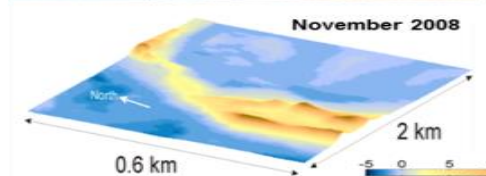

(b)

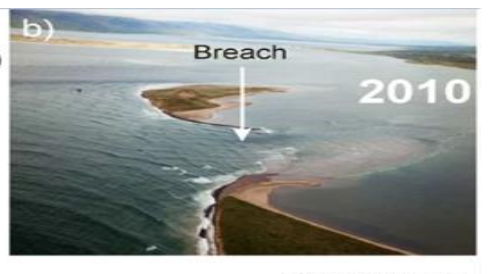

(d)

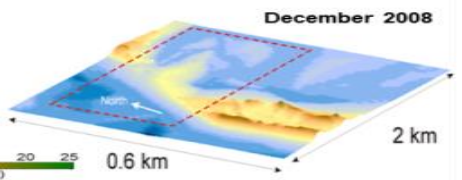

(e)

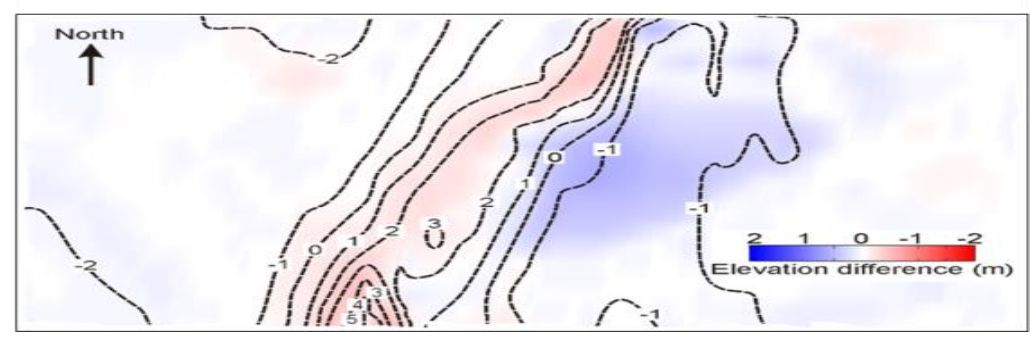

Figure 3. Oblique aerial view of Rossbeigh Spit (a) pre-breach c. 2003; and (b) post-breach c. 2010. XBeach DTM: (c) pre-breach 10/2008; and (d) post-breach 12/2008. (e) Difference between measured and predicted bathymetry / topography for the breach region (defined by the red rectangle in d) 
In this case, the methods used to provide missing information required to set up and calibrate the model were verified by the model results which showed good quantitative and qualitative agreement with observational data. However, the example does highlight a problem frequently encountered in morphological modelling regarding the quantity and quality of data available to build, calibrate and validate the model. While efforts to improve morphological models are applauded, equal attention must be given to these aspects of the modelling process.

\section{Hopton-on-Sea, England}

In response to a storm in March 2013 that resulted in beach lowering and cliff recession of $c .5 \mathrm{~m}$ along a $110 \mathrm{~m}$ frontage at Hopton-on-Sea, England, hydrodynamic and morphological modelling was used to assist in the design of a new coastal protection scheme. The scheme must provide acceptable level of protection and, to be granted consent, no adverse environmental impact must be demonstrated. The study used MIKE by DHI and XBeach to examine two proposed schemes: (a) three large fishtail rock groynes (Scheme 1) and; (b) ten 'double-head' curved rock groynes (Scheme 2). Although it is common practice and cost-effective to use only one numerical model, if agreement can be established between predictions from two or more morphological models, uncertainty in the model results can be reduced and greater confidence provided.

As a illustration of model results, Figure 4 shown the average of the changes in bed elevation predicted by MIKE and 2D XBeach for north-easterly wave conditions (significant wave height, $H_{s}=2.0 \mathrm{~m}$, peak wave period, $T_{p}=8 \mathrm{~s}$ ) for (a) Scheme 1; and (b) Scheme 2. While it is unavoidable not to be able to verify model results in studies of this nature until the scheme is built and functioning, modelling uncertainty is reduced and confidence is provided by the good agreement between different models. The MIKE and XBeach model results assisted in the design of defence scheme structures (height, profile, length and spacing) and have provided guidance on how the scheme is likely to perform and the impacts it may have on the adjacent shorelines. They have also allowed preliminary material quantities and cost estimates to be confirmed with greater confidence. Together, the model results have assisted the development of an improved final scheme design which minimizes potential environmental impacts. 
(a)

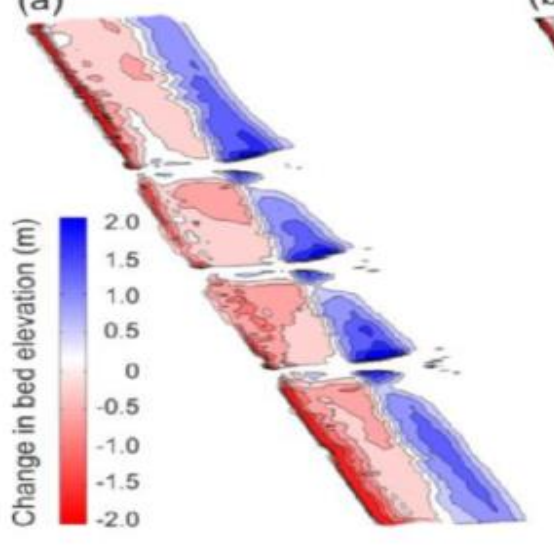

(b)

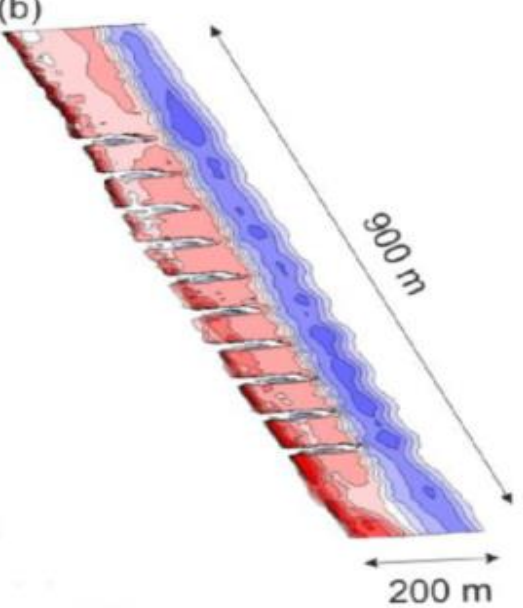

Figure 4. Average of the changes in bed elevation predicted by MIKE and 2D XBeach for northeasterly wave conditions (significant wave height, $H_{s}=2.0 \mathrm{~m}$, peak wave period, $T_{p}=8 \mathrm{~s}$ ) for (a) Scheme 1; and (b) Scheme 2.

\section{Conclusions}

The modelling of coastal morphodynamics over decadal and centennial timescales must account for ecological and human influences as well as the better understood physical processes.

The establishment of a conceptual understanding of local sediment transport and morphodynamic behaviour provides the hypothesis to test subsequent model performance and help in understanding the magnitudes and directions of sediment transport and the associated rate and scale of morphological changes.

In practice forcing terms, processes and coastal systems responses cannot be determined beyond certain time- and space-scales and models should not be pushed beyond these limits.

If distinct morphological features define the character of a system attempts should first be made to describe the behaviour of features as simple as possible before attempting to simulate their formation and behaviour together.

Morphological thresholds, which can cause large variations in the physical to occur from small modifications of the inputs (e.g. catastrophic behaviour) and may not be predicted by the model or detected by sensitivity analyses. To date this aspect of morphological modelling has been scarcely addressed and urgently requires attention. 
Compromise must be reached between the end-user requirements for a given morphological model and the ability of that model to deliver the results required.

\section{Acknowledgements}

Thanks are extended to Bourne Leisure Ltd. who funded the work at Hopton-onSea. Jon Williams and Thomas Conduché were supported by the Ports, Coastal and Offshore Group at Mott MacDonald. Luciana Esteves thanks the Bournemouth University Fusion Funds that facilitated her contribution to this paper and the support to attend the conference.

\section{References}

Cea, L., and French, J. R. (2012). "Bathymetric error estimation for the calibration and validation of estuarine hydrodynamic models". Estuarine, Coastal and Shelf Science, 100, 124-132.

Chu, K., Winter, C., Hebbeln, D. and Schulz, M. (2013). "Improvement of morphodynamic modeling of tidal channel migration by nudging". Coastal Engineering, 77, 1-13.

Mayo, T., Butler, T., Dawson, C., and Hoteit, I. (2014). "Data assimilation within the Advanced Circulation (ADCIRC) modeling framework for the estimation of Manning's friction coefficient". Ocean Modelling, 76, 43-58.

Margvelashvili, N. and Campbell, E. (2012). "Sequential data assimilation in fine resolution models using error subspace emulators: theory and preliminary evaluation”. Journal of Marine Systems, 90, 13-22.

Margvelashvili, N., Andrewartha, J., Herzfeld, M., Robson, B. J. and Brando, V. E. (2013). "Satellite data assimilation and estimation of a 3D coastal sediment transport model using error-subspace emulators". Environmental Modelling and Software, 40, 191-201.

Mossman, H. L., Davy, A. J., \& Grant, A. (2012). "Does managed coastal realignment create saltmarshes with 'equivalent biological characteristics' to natural reference sites?". Journal of Applied Ecology, 49(6), 1446-1456.

Kirwan, M. L., and Murray, A. B. (2007). "A coupled geomorphic and ecological model of tidal marsh evolution". Proceedings of the National Academy of Sciences, 104(15), 6118-6122. 
Kristensen, S. E., Drønen, N., Deigaard, R. and Fredsoe, J. (2013). "Hybrid morphological modelling of shoreline response to a detached breakwater". Coastal Engineering, 71, 13-27.

Refsgaard, J. C., Madsen, H., Andréassian, V., Arnbjerg-Nielsen, K., Davidson, T. A., Drews, M., Hamilton, D. P. \& Christensen, J. H. (2014). "A framework for testing the ability of models to project climate change and its impacts". Climatic change, 122(1-2), 271-282.

Scott, T. R. and Mason, D. C. (2007). "Data assimilation for a coastal area morphodynamic model: Morecambe Bay”. Coastal Engineering, 54(2), 91-109.

Serafy, G., Eleveld, M., Blaas, M., van Kessel, T., Aguilar, S. and van der Woerd, H. (2011). Improving the description of the suspended particulate matter concentrations in the southern North Sea through assimilating remotely sensed data. Ocean Science Journal, 46(3), 179-204.

Smith, P. J., Thornhill, G. D., Dance, S. L., Lawless, A. S., Mason, D. C., and Nichols, N. K. (2013). "Data assimilation for state and parameter estimation: application to morphodynamic modelling". Quarterly Journal of the Royal Meteorological Society, 139(671), 314-327.

Splinter, K. D., Turner, I. L., and Davidson, M. A. (2013). "How much data is enough? The importance of morphological sampling interval and duration for calibration of empirical shoreline models". Coastal Engineering, 77, 14-27.

Temmerman, S., Meire, P., Bouma, T. J., Herman, P. M., Ysebaert, T., \& De Vriend, H. J. (2013). "Ecosystem-based coastal defence in the face of global change". Nature, 504(7478), 79-83.

Turner, R. K., Adger, W. N., Lorenzoni, I., \& Bateman, I. J. (1998). "Towards integrated modelling and analysis in coastal zones: principles and practice" (No. 11). LOICZ Core Project Office, Netherlands Institute for Sea Research (NIOZ).

Williams, J. J., Esteves L. S. \& Rochford, L. A. (2015). "Modelling Storm Responses on a High-Energy Coastline with XBeach". Modelling Earth Systems and Environment (submitted). 